\title{
Erratum to: Development of a knowledge assessment tool for dermatotoxicity caused by inhibitors of epidermal growth factor receptor
}

Dimitrios Konstantinos Papageorgiou • Theocharis Konstantinidis •

Nektaria Skandalaki • Anna Papadouri • Theodora Pappa •

Stavroula Poulopoulou

Published online: 29 August 2013

(C) Springer-Verlag Berlin Heidelberg 2013

Erratum to: Support Care Cancer (2012) 20:1667-1671

DOI 10.1007/s00520-011-1257-y

The original version of this article unfortunately contained spelling error. The name of "Theocharis Konstantinidis" is now corrected in the author group of this article.

The online version of the original article can be found at http://dx.doi.org/ 10.1007/s00520-011-1257-y.

D. K. Papageorgiou ( $\square)$

Euroclinic Athens,

Athens, Greece

e-mail: dimpapa75@yahoo.gr

T. Konstantinidis

Department of Nursing, Technological Institute of Heraklion,

Heraklion, Greece

e-mail: theokons@hotmail.com

N. Skandalaki

Venizeleio Hospital of Heraklion,

Heraklion, Greece

e-mail: dimtery@yahoo.com

A. Papadouri · T. Pappa

Oncology Hospital Ag. Anargiri,

Athens, Greece

A. Papadouri

e-mail: annapapadouri@windowslive.com

T. Pappa

e-mail: d.pappa@mail.gr

S. Poulopoulou

Department of Statistics,

Athens University of Economics and Business,

Athens, Greece

e-mail: spoulopo@aueb.gr 